EPJ Web of Conferences 78, 01009 (2014)

DOI: $10.1051 /$ epjconf / 20147801009

(C) Owned by the authors, published by EDP Sciences, 2014

\title{
Wigner crystals: New realizations of an old idea
}

\author{
J. Sólyom ${ }^{1,2, a}$ \\ ${ }^{1}$ Wigner Research Centre for Physics, Institute for Solid State Physics and Optics, Budapest, Hungary \\ ${ }^{2}$ Department of Physics, Roland Eötvös University, Budapest, Hungary
}

\begin{abstract}
In this review, after a brief discussion of Wigner's ideas about the localization of electrons due to Coulomb interaction in a low-density electron system with neutralizing background and the formation of a periodic array, we present the various possibilities to realize Wigner crystals: on the surface of liquid helium, in semiconductor heterostructures, in graphene, in 1D systems and the analogous crystalline states in dusty plasma or in the assembly of trapped ions.
\end{abstract}

\section{Introduction}

The second half of the twenties of the last century was a fascinating period in the development of quantum mechanics. A year after the publication of W. Heisenberg's paper on matrix mechanics in 1925, and in the same year that A. Schrödinger's paper presenting his famous equation appeared, E. Fermi and P. A. M. Dirac pointed out that particles subject to the Pauli exclusion principle obey a special statistics, called Fermi-Dirac statistics since then. One more year later A. Sommerfeld applied this theory to electrons in metals, and explained a number of properties of simple metals, even though the interaction between electrons was neglected in that model. The first attempt to take interactions into account appeared next year when D. R. Hartree used a mean-field-like approach to incorporate the effect of other electrons in the one-particle Schrödinger equation. Finally, in 1930, V. Fock showed that the Pauli principle leads to an exchange correction to the energy eigenvalues.

That was the situation in the thirties when E. Wigner and his student, F. Seitz started to work on the cohesive energy of metals. They recognized that the corrections beyond Hartree-Fock, dubbed correlation energy by them, are important to understand the metallic bond. Wigner then asked the question $[1,2]$ whether at low electron densities, where the Coulomb energy is much larger than the kinetic energy, the metallic state would survive or the electrons become localized and form a periodic crystalline array. This then led him to propose the concept of electron crystal.

\section{The original idea}

We consider a system of electrons of density $n_{\mathrm{e}}$. Assigning a Wigner-Seitz sphere to each electron, its radius is

$$
r_{0}= \begin{cases}(3 / 4 \pi)^{1 / 3} n_{\mathrm{e}}^{-1 / 3} & \text { in 3D, } \\ (1 / \pi)^{1 / 2} n_{\mathrm{e}}^{-1 / 2} & \text { in 2D. }\end{cases}
$$

\footnotetext{
ae-mail: solyom.jeno@wigner.mta.hu
} 
The properties of the system are determined by the competition of the kinetic and potential energies. The kinetic energy per particle is given approximately by the Fermi energy. The Fermi momentum is inversely proportional to the mean distance between electrons, and so

$$
E_{\text {kin }} / N_{\mathrm{e}} \approx \frac{\hbar^{2} k_{\mathrm{F}}^{2}}{2 m} \propto r_{0}^{-2} \propto n_{\mathrm{e}}^{2 / D} .
$$

On the other hand, the potential energy per particle due to the Coulomb interaction can be approximated by the Coulomb energy between neighbouring electrons,

$$
E_{\mathrm{pot}} / N_{\mathrm{e}} \approx \frac{e^{2}}{4 \pi \epsilon \epsilon_{0} r_{0}} \propto n_{\mathrm{e}}^{1 / D} .
$$

The parameter characterizing the correlations between the particles is then

$$
\Gamma=\frac{E_{\text {pot }}}{E_{\text {kin }}} \propto n_{\mathrm{e}}^{-1 / D} .
$$

When $\Gamma$ is much less than unity, the interaction between electrons is negligible compared to the kinetic energy and the system behaves as a nearly free electron gas. Wigner conjectured that in the opposite case, when the Coulomb potential dominates, the electrons might be localized in a crystalline structure. This can happen in the low-density limit, $n_{\mathrm{e}} \ll 1$, and this state is called Wigner crystal (WC). Quantum mechanically the electrons oscillate around their equilibrium positions even in the ground state. The crystal melts if the root mean square deviation from the equilibrium position becomes comparable to the lattice constant of the Wigner crystal. A naive calculation in the harmonic approximation gives $r_{0}>20 a_{0}$ for the stability region of the crystalline state, where $a_{0}$ is the Bohr radius.

Better calculations can be done for the interacting electron system in a uniform background using Monte Carlo methods. The most recent calculations on the three-dimensional electron gas indicate that the body-centered cubic Wigner crystal is stable only if $r_{0} / a_{0}>106$ [3]. The numerically determined phase diagram [4] is shown in Fig. 1. As one sees, the transition from the homogeneous liquid-like phase to the Wigner crystal phase is not direct at low temperatures. A partially polarized fluid appears first as the density is lowered, and an antiferromagnetic crystalline state may exist in a narrow range of densities.

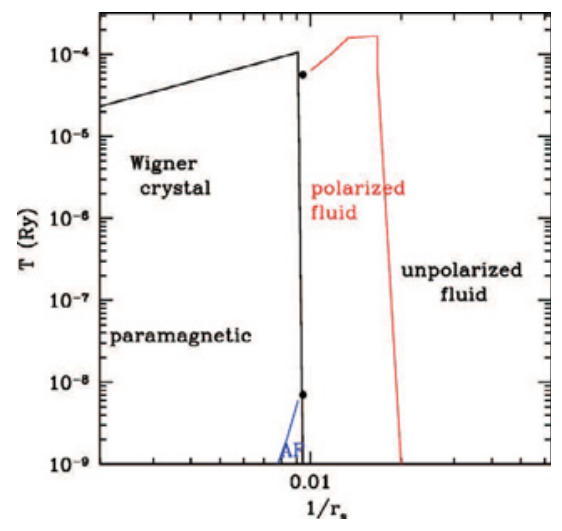

Figure 1. Numerically determined phase diagram of the three-dimensional interacting electron system [L. Candido, B. Bernu and D. M. Ceperley, Phys. Rev. B 70, 094413 (2004)]

The normal metallic electron densities correspond to the range $2<r_{0} / a_{0}<6$. Hence the kinetic energy is the dominant term, $\Gamma$ is small, and the electrons are delocalized in normal metals. An 
extremely low electron density would be needed to satisfy the condition for the existence of Wigner crystal, and therefore practically there is no hope to observe a 3D crystalline arrangement of electrons. The stability condition of the Wigner crystal is, much less restrictive for a $2 \mathrm{D}$ electron system. According to the most recent calculations [5] an antiferromagnetic triangular Wigner crystal is the most stable state for $r_{0} / a_{0}>31$, which transforms into a ferromagnetic Wigner crystal for $r_{0} / a_{0}>38$.

\section{Classical Wigner crystal on the surface of liquid helium}

Although Wigner crystal could be formed easier in 2D electron systems, it took more than forty years until the first experimental realization of a Wigner crystal was achieved in a classical, 2D Coulomb system, namely in a monolayer of electrons trapped in a potential well above the surface of liquid helium [6]. In this case the kinetic energy of electrons is fixed by the temperature, $E_{\mathrm{kin}} / N_{\mathrm{e}} \approx k_{\mathrm{B}} T$, hence the parameter $\Gamma$, which is now

$$
\Gamma \approx e^{2} /\left(4 \pi \epsilon_{0} r_{0} k_{\mathrm{B}} T\right)=e^{2} \pi^{1 / 2} n_{\mathrm{e}}^{1 / 2} /\left(4 \pi \epsilon_{0} k_{\mathrm{B}} T\right),
$$

could be conveniently varied by varying the areal density in the range $10^{5} \mathrm{~cm}^{-2}<n_{\mathrm{e}}<10^{9} \mathrm{~cm}^{-2}$. Contrary to the quantum case, $\Gamma$ is now proportional to the square root of the electron density, and Wigner crystal appears as the density is increased. Unfortunately we have no appropriate tool to directly observe the formation of the Wigner crystal as more and more electrons are added to the surface layer. In the classic experiment by Grimes and Adams the resonance in the surface impedance of the radio-frequency field due to excitation of surface capillary waves (ripplons) on the surface of helium coupled to the vibrations of the Wigner lattice was detected. The experiments gave a clear indication of the formation of a Wigner crystal for high densities, at $\Gamma \approx 137 \pm 15$, in reasonable agreement with the expectations based on Monte Carlo calculations [7].

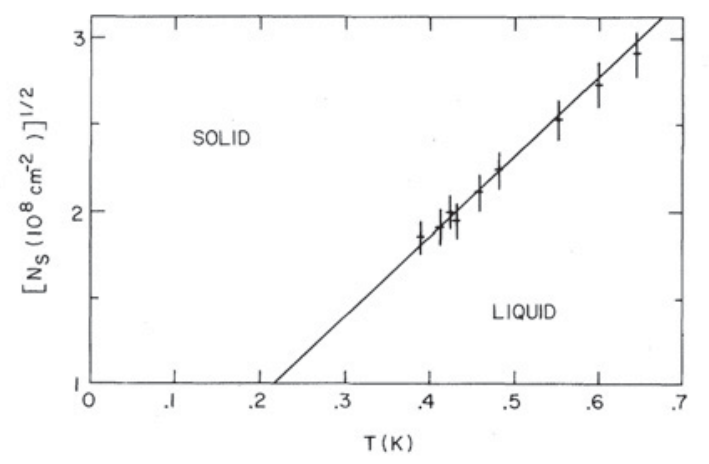

Figure 2. The measured electron-liquid to electron-crystal phase boundary in the areal density versus temperature plane for the two-dimensional electron system trapped on the surface of liquid helium [C. C. Grimes and G. Adams, Phys. Rev. Lett. 42, 795 (1979)]

\section{Wigner crystal in semiconductor heterostructures}

At the densities used in the previous experiment, the electrons behave classically. Quantum Wigner crystal might be observed at higher densities in the $2 \mathrm{D}$ electron or hole gas created in high-mobility semiconductor heterostructures, where the electron or hole density can be varied by a bias voltage. In fact, measurements of the low-temperature resistivity of GaAs/GaAlAs heterostructures [8] indicate a metal-insulator transition in the ground state at the hole density $7.7 \times 10^{9} \mathrm{~cm}^{-2}$ as the carrier density is varied. This critical density corresponds to $r_{0} / a_{0} \approx 35$, which is rather close to the value predicted 
by the numerical calculations for the 2D Coulomb system. Therefore the metal-insulator transition and the insulating behaviour at low carrier densities have been interpreted as being due to Wigner crystallization, although Anderson localization due to disorder could not definitely be ruled out.

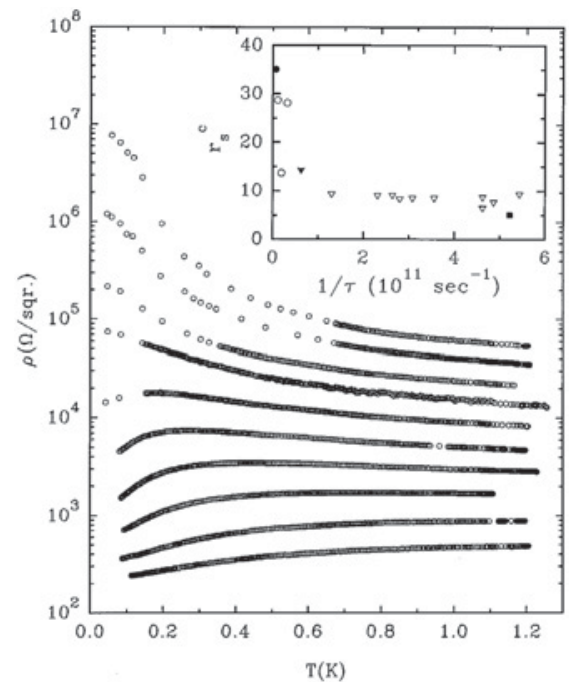

Figure 3. Temperature dependence of the resistivity of the 2D hole system in GaAs/ $\mathrm{Al}_{x} \mathrm{Ga}_{1-x}$ As for different hole densities ranging from $4.8 \times 10^{9} \mathrm{~cm}^{-2}$ till $3.72 \times 10^{9} \mathrm{~cm}^{-2}$ [J Yoon, C. C. Li, D. Shahar, D. C. Tsui and M. Shayegan, Phys. Rev. Lett. 82, 1744 (1999)]

It has been proposed $[9,10]$ that the localization of electrons and the formation of Wigner crystal might be facilitated in the $2 \mathrm{D}$ electron gas by a strong perpendicular magnetic field, where the electrons are forced into cyclotron orbits with decreasing radius for increasing field. The kinetic energy is reduced in the quantized states on Landau levels, and the case, when only the lowest Landau level is partially filled, might be most promising. Crystallization may occur when the magnetic length $l_{0}=\sqrt{\hbar / e B_{\perp}}$ is small compared to the typical interparticle spacing. In this case, however, the other state with which the Wigner crystal has to compete is not a conventional homogeneous electron liquid state, but the incompressible Laughlin state of the fractional quantum Hall (FQH) liquid. There is strong evidence from numerical calculations [11-17] that the energy of the Wigner crystal is lower than that of the Laughlin state for Landau level filling $v<1 / 7$, which implies that the FQH effect can be observed at higher fillings only, although, as can be seen in Fig. 4, the energy difference between the Wigner crystal and the Laughlin state is quite small.

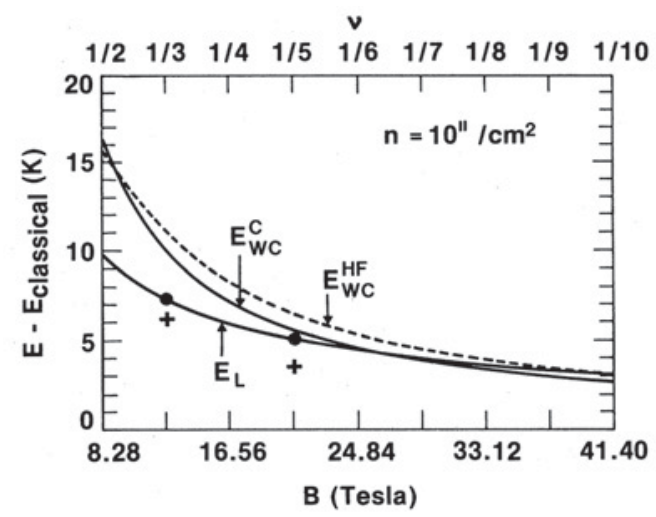

Figure 4. Comparison of the energies of the Wigner crystal and of the Laughlin state in strong magnetic fields for a fixed areal density of electrons [P. K. Lam and S. M. Girvin, Phys. Rev. B 30, 473 (1984)] 


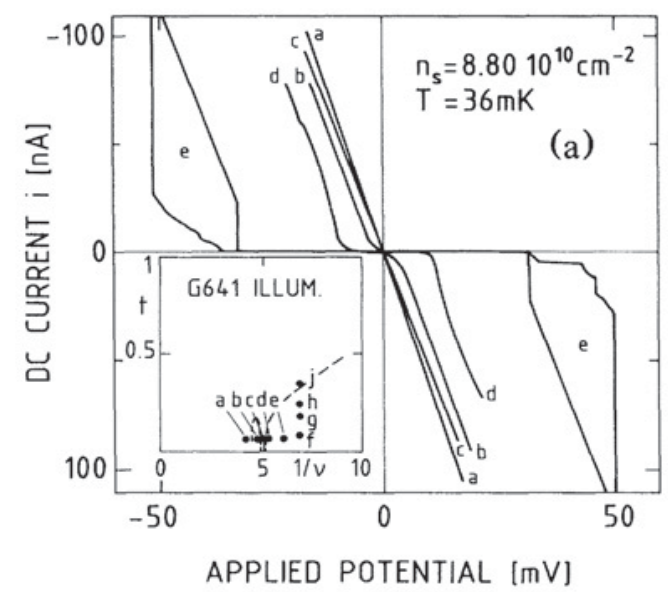

Figure 5. Current-voltage characteristics of a $\mathrm{GaAs} / \mathrm{GaAlAs}$ heterojunction at very low temperature $(T=36 \mathrm{mK})$ in fields ranging from $15 \mathrm{~T}$ till $22 \mathrm{~T}[\mathrm{~F}$. I. B. Williams et al., Phys. Rev. Lett. 66, 3285 (1991)]

The earliest experimental indication for ordering of electrons into a quantum Wigner crystal was obtained from high-magnetic-field transport measurements [18]. Since the electron lattice is pinned by the disorder potential, current can flow only when the electric field exceeds a threshold value where the lattice is depinned. This effect gives rise to a nonlinear conductivity and activated temperature dependence which have been observed in several experiments [19-22]. Radio-frequency spectroscopy detected the onset of transverse gapless magnetophonons [23] roughly at the same fields where the nonlinearity appears. A pinning resonance of the electron solid - interpreted as the Wigner crystal pinning mode, that is oscillations of pinned crystalline domains in the disorder potential - could also be observed in the microwave spectra at low temperatures [24, 25]. Of course the melting temperature of the solid phase depends on the electron density and on the magnetic field, but it was found that in fact only the ratio $n_{\mathrm{e}} / B$, that is the filling factor of the Landau level, $v=n_{\mathrm{e}} h / e B$ matters [25]. A detailed review of the theoretical and experimental works on 2D Wigner crystals and in particular on magnetic-field-induced 2D Wigner crystals can be found in [26, 27].

All these together give a rather strong evidence for the existence of a magnetic-field-induced quantum electron solid. Moreover it could be concluded from the experiments that - in agreement with the theoretical predictions - the 2D electron system in strong magnetic fields is an insulating solid if the filling factor of the lowest Landau level $v<0.22$ except for $v=0.2[28,29]$ where the ground state is a Laughlin state and the fractional quantum Hall effect is observed. A reentrant behaviour is found in increasing (or decreasing) magnetic field when the filling factor passes through $v=1 / 5$. While the off-diagonal component of the resistivity, $\rho_{x y}$, varies smoothly around $v=1 / 5$, the diagonal component, $\rho_{x x}$, shows a dramatic, exponential increase at low temperatures on both sides of $v=1 / 5$ filling as the temperature is lowered. However, at $v=1 / 5$, in the FQH state, $\rho_{x x}$ vanishes.

This can be understood in the following way. In a 2D system the components of the conductivity tensor are related to the resistivity as

$$
\sigma_{x x}=\frac{\rho_{x x}}{\rho_{x x}^{2}+\rho_{x y}^{2}}, \quad \sigma_{x y}=\frac{\rho_{x y}}{\rho_{x x}^{2}+\rho_{x y}^{2}} .
$$

Both the FQH and the WC state are insulating, $\sigma_{x x}=0$, but this is achieved in different ways in the two systems. While $\rho_{x y}$ is finite and smooth across the WC-FQH boundary, $\rho_{x x}=0$ as $T \rightarrow 0$ at $v=1 / 5$ in the FQH liquid. On the other hand the vanishing of $\sigma_{x x}$ in the Wigner crystal is due to the divergence of $\rho_{x x}$ to infinity as $T \rightarrow 0$. 
EPJ Web of Conferences

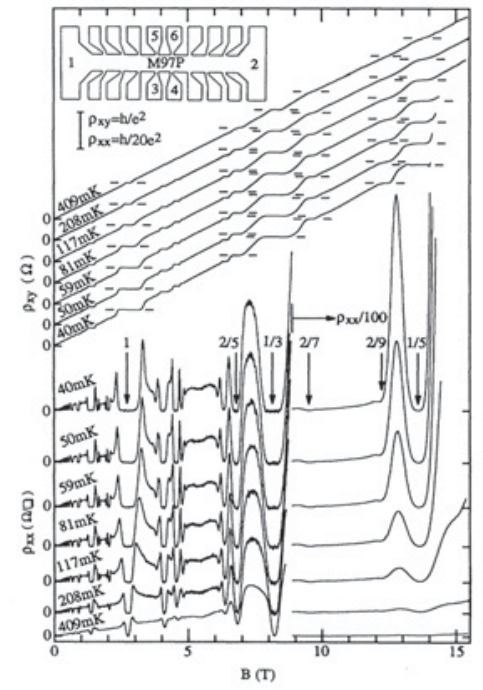

Figure 6. Field dependence of the resistivity tensor components $\rho_{x x}$ and $\rho_{x y}$ for different temperatures in a GaAs/GaAlAs sample in strong magnetic fields [T. Sajoto, Y. P. Li, L. W. Engel, D. C. Tsui and M. Shayegan, Phys. Rev. Lett. 70, 2321 (1993)]

\section{Bilayer quantum Wigner crystal, bubbles and stripes}

In a wider quantum well the electrons can be arranged in two layers and new incompressible FQH liquid states may appear at even denominator filling factors such as $v=1 / 2$. Resistivity measurements [30] revealed insulating phases on both sides of this filling, resembling the situation we have seen near $v=1 / 5$, and these states have been interpreted as pinned, correlated bilayer Wigner crystals [31, 32].

In weaker magnetic fields, where electrons occupy higher Landau levels, the FQH liquid state gets unstable. Hartree-Fock calculations indicate that Wigner crystal can still exist at low electron or hole fillings of the second lowest or of a higher Landau level when the interparticle distance is small compared to the magnetic length. When the lattice constant becomes comparable to the magnetic length, bubbles with more than one electron per site or stripes are formed [33,34]. This has been corroborated first by density-matrix renormalization-group (DMRG) calculations and later experimentally, too. According to numerical works [35], Wigner crystal can exist when the filling factor of a higher Landau level is less than about 0.2 , while stripe phase appears for nearly half filled Landau levels. In the intermediate regime bubble phases are stable. The experimentally observed microwave resonances in the real part of the frequency dependent diagonal conductivity close to integer fillings were interpreted as coming from a Wigner crystal state [36]. A resonance at a different frequency appears when the bubble phase appears with a first-order transition [37].

\section{Wigner crystal in graphene}

Graphene is an ideal two-dimensional system. Contrary to the $2 \mathrm{D}$ electron system in a heterojunction, the dispersion relation of electrons in graphene is linear around the Dirac points. Therefore the kinetic and potential energies scale identically with the density, hence the $\Gamma$ parameter is independent of the density, and Wigner crystal cannot be formed without magnetic field. Wigner crystal may, however, exist at low fillings of the lowest Landau level in strong fields [38]. When higher Landau levels are partially filled, bubble phases with two or three particles per lattice site and stripe phases have been predicted both from analytic (Hartree-Fock) and numerical calculations [38, 39]. 


\section{Wigner crystal in 1D systems}

Until now we considered 2D systems, since quantum fluctuations destroy long-range positional order in one dimension. Wigner crystals may, nevertheless, exist in physically 1D systems, such as carbon nanotubes or quantum wires. The unusual single-electron transport behaviour of carbon nanotubes in magnetic fields, the shift of the Coulomb blockade peaks with field could be accounted for by assuming Wigner crystallization [40]. Similarly, the low-temperature transport properties of quantum wires suggest the formation of a $1 \mathrm{D}$ electron chain at low densities. As the electron density increases and the confinement weakens, this linear arrangement is transformed into a zigzag structure before more rows are formed [41]. The physics of Wigner crystals in quantum wires has been discussed in [42].

\section{Wigner crystal versus Yukawa or Coulomb crystal}

The simplest model for Wigner crystals is that of electrons in a uniform neutralizing background. Alternatively we could consider a one-component plasma of positively charged particles immersed in a uniform background. Physical realizations are - among others - ions in an ion trap, or on macroscopic scales colloidal particles in aqueous solutions or dust particles in a plasma. It is quite natural to expect that in the same way as for electrons, the Coulomb interaction may order the positively charged particles, if the $\Gamma$ parameter is large enough. They are then called Coulomb crystal, colloidal crystal or Yukawa crystal if the screening of the Coulomb interaction is essential and Yukawa potential has to be used to describe the properties. It should be noted that quantum effects can usually be neglected, and these systems are analogous to classical Wigner crystals. Direct observation of the crystalline structure is possible in these cases. Bragg scattering consistent with a body-centered cubic arrangement of the ions has indeed been observed, e.g., when a large enough number $\left(10^{5}\right)$ of ${ }^{9} \mathrm{Be}^{+}$ions were trapped in a Penning trap [43], while optical experiments revealed crystalline structure even when the number of ions was below a thousand [44]. Regular patterns were observed in dusty plasma as well [45].

\section{Summary}

Almost half a century after its prediction, the technical developments in producing low-density electron systems allowed the unequivocal demonstration of the existence of quantum Wigner crystals in two dimensions, especially in strong magnetic fields. We have strong indications for the formation of Wigner crystal in quasi-1D systems as well. The newly discovered ideal two-dimensional system, graphene, with its Dirac cone excitation spectrum, presents a new challenge to find experimentally the Wigner crystal phase, which, in this system may exist in strong fields only. The study of the properties of the analogous Coulomb crystal of ions and Yukawa crystal in plasma may also help to better understand classical Wigner crystals.

\section{References}

[1] E. Wigner, Phys. Rev. 46, 1002 (1934)

[2] E. Wigner, Trans. Faraday Soc. 34, 678 (1938)

[3] N. D. Drummond et al., Phys. Rev. B 69, 085116 (2004)

[4] L. Candido, B. Bernu and D. M. Ceperley, Phys. Rev. B 70, 094413 (2004)

[5] N. D. Drummond and R. J. Needs, Phys. Rev. Lett. 102, 126402 (2009)

[6] C. C. Grimes and G. Adams, Phys. Rev. Lett. 42, 795 (1979) 
[7] R. C. Gann, S. Chakravarty and G. V. Chester, Phys. Rev. B 20, 326 (1979)

[8] J Yoon, C. C. Li, D. Shahar, D. C. Tsui and M. Shayegan, Phys. Rev. Lett. 82, 1744 (1999)

[9] Yu. E. Lozovik and V. I. Yudson, JETP Lett. 22, 11 (1975)

[10] H. Fukuyama, Solid State Commun. 19, 551 (1976)

[11] K. Maki and X. Zotos, Phys. Rev. B 28, 4349 (1983)

[12] P. K. Lam and S. M. Girvin, Phys. Rev. B 30, 473 (1984)

[13] D. Levesque, J. J. Weis and A. H. MacDonald, Phys. Rev. B 30, 1056(R) (1984)

[14] K. Esfarjani and S. T. Chui, Phys. Rev. B 42, 10758 (1990)

[15] X. Zhu and S. G. Louie, Phys. Rev. Lett. 70, 335 (1993)

[16] R. Price, P. M. Platzman and S. He, Phys. Rev. Lett. 70, 339 (1993)

[17] K. Yang, F. D. M. Haldane and E. H. Rezayi, Phys. Rev. B 64, 081301(R) (2001)

[18] E. E. Mendez, M. Heiblum, L. L. Chang and L. Esaki, Phys. Rev. B 28, 4886 (1983)

[19] R. L. Willett et al., Phys. Rev. B 38, 7881 (1988)

[20] V. J. Goldman, M. Santos, M. Shayegan and J. E. Cunningham, Phys. Rev. Lett. 65, 2189 (1990)

[21] F. I. B. Williams et al., Phys. Rev. Lett. 66, 3285 (1991)

[22] M. A. Paalanen et al., Phys. Rev. B 45, 13784 (1992)

[23] E. Y. Andrei et al., Phys. Rev. Lett. 60, 2765 (1988)

[24] P. D. Ye et al., Phys. Rev. Lett. 89, 176802 (2002)

[25] Y. P. Chen et al., Nature Physics 2, 452 (2006)

[26] Yu. P. Monarkha and V. E. Syvokon, Low Temp. Phys. 38, 1067 (2012)

[27] M. Shayegan, in Perspectives in Quantum Hall Effets, Edited by S. Das Sarma and A. Pinczuk, (John Wiley \& Sons, New York, 1997) p. 343

[28] H. W. Jiang et al., Phys. Rev. Lett. 65, 633 (1990)

[29] T. Sajoto, Y. P. Li, L. W. Engel, D. C. Tsui and M. Shayegan, Phys. Rev. Lett. 70, 2321 (1993)

[30] H. C. Manoharan, Y. W. Suen, M. B. Santos and M. Shayegan, Phys. Rev. Lett. 77, 1813 (1996)

[31] L. Zheng and H. A. Fertig, Phys. Rev. B 52, 12282 (1995)

[32] S. Narasimhan and T.-L. Ho, Phys. Rev. B 52, 12291 (1995)

[33] M. M. Fogler, A. A. Koulakov and B. I. Shklovskii Phys. Rev. B 54, 1853 (1996)

[34] M. M. Fogler, in High Magnetic Fields, Applications in Condensed Matter Physics and Spectroscopy LNP 595, C. Berthier, L. P. Lévy, G. Martinez (Eds.) (Springer Berlin, 2002) p. 98

[35] N. Shibata and D. Yoshioka, Phys. Rev. Lett. 86, 5755 (2001)

[36] Y. Chen et al., Phys. Rev. Lett. 91, 016801 (2003)

[37] R. M. Lewis et al., Phys. Rev. Lett. 93, 176808 (2004)

[38] C.-H. Zhang and Y. N. Joglekar, Phys. Rev. B 75, 245414 (2007)

[39] H. Wang, D. N. Sheng, L. Sheng and F. D. M. Haldane, Phys. Rev. Lett. 100, 116802 (2008)

[40] V. V. Deshpande and M. Bockrath, Nature Phys. 4, 314 (2008)

[41] W. K. Hew et al., Phys. Rev. Lett. 102, 056804 (2009)

[42] J. S. Meyer and K. A. Matveev, J. Phys.: Condens. Matter 21, 023203 (2009)

[43] J. N. Tan, J. J. Bollinger, B. Jelenkovic and D. J. Wineland, Phys. Rev. Lett. 75, 4198 (1995)

[44] A. Mortensen, E. Nielsen, T. Matthey and M. Drewsen, Phys. Rev. Lett. 96, 103001 (2006)

[45] A. Melzer, Phys. Rev. E 67, 016411 (2003) 